\title{
Contrast transfer characteristics of the light sword optical element designed for presbyopia compensations
}

\section{K. Petelczyc}

\section{S. Bará}

\section{A. Ciro López}

\section{Z. Jaroszewicz mmtzjaroszewicz@post.pl}

\section{K. Kakarenko}

\section{A. Kolodziejczyk}

\section{Sypek}

Faculty of Physics, Warsaw University of Technology, Koszykowa 75, 00-662 Warsaw, Poland

Area de Optica, E.U., Optica e Optometria, Universidade de Santiago de Compostela, 15782 Santiago de Compostela, GALIZA Spain

Grupo de Optica y Fotonica, Instituto de Fisica, Universidad de Antioquia, A.A1226, Medellin, Colombia

Facultad de Ciencias, Instituto Tecnologico Metropolitano, calle 73 \#76A-354, Medellin, Colombia Institute of Applied Optics, Kamionkowska 18, 03-805 Warsaw, Poland; National Institute of Telecommunications, Szachowa 1, 04-894 Warsaw, Poland

Faculty of Physics, Warsaw University of Technology, Koszykowa 75, 00-662 Warsaw, Poland

Faculty of Physics, Warsaw University of Technology, Koszykowa 75, 00-662 Warsaw, Poland

The paper discusses the abilities of the light sword optical element (LSOE) for presbyopia compensation. The imaging properties are analyzed by means of the modulation transfer functions and output images of the star resolution test. All numerical calculations are performed assuming an optical set-up simulating the presbyopic human eye with $3 \mathrm{~mm}$ pupil acting on-axis and based on the Gullstrand model. In order to have a meaningful comparison we expand our study and present adequate analysis for other elements potentially useful in ophthalmology as reading glasses, bifocal lenses and axicons. According to the obtained results the LSOE can successfully realize vision with an extended depth of field. Under assumed conditions the element makes possible the compensation of an assumed defocus up to 4 dioptres. The output images formed by the LSOE are well recognizable and have acceptable qualities for near as well as far object distances. [DOI: http://dx.doi.org/10.2971/jeos.2011.11053]

Keywords: diffraction, presbyopia, vision, image formation, extended depth of field

\section{INTRODUCTION}

Presbyopia is probably the most common defect of the aging human eye. The majority of the population suffers this inconvenience. The accommodation ability of a human eye's lens decreases constantly from above $10 \mathrm{D}$ at a newborn to about $1 \mathrm{D}$ when be getting on for fifty $[1,2,3]$. Therefore there are conducted intensive investigations searching for effective corrections of presbyopia [4]. Among various proposals the light sword optical element (LSOE) [5] was recently studied as a promising alternative and it was shown that its Strehl ratio shows a significant superiority in terms of uniformity within the prescribed range of optical power to be covered comparing with other optical elements designed especially for presbyopia compensation [6]. According to the obtained results the LSOE can be a particularly promising tool for a substitution of the accommodation process. The Strehl coefficients corresponding to the LSOE with fixed aperture diameter have substantial and almost constant values.

However, one should bear in mind that the Strehl ratio gives only a very general insight into imaging and quality of its performance in ophthalmology was discussed [7, 8, 9]. Then an effective image assessment based exclusively on this method is considerably limited. Therefore it seems to be justified to investigate the imaging properties of the LSOE in a more detailed way in order to gain more proofs of the LSOE applicability for presbyopia compensation. This work presents results based on the analysis of modulation transfer functions (MTFs) and images of the star resolution test (Siemens star). This approach enables to estimate the contrast transfer and the quality of the output images depending on the structure of the input objects. In order to maintain consistence with the results presented in Ref. [6], the optical set-up equivalent to the simplified Gullstrand model of the human eye was still assumed $[10,11]$ and we used the same software package based on the convolution method $[12,13]$. Our studies are concentrated on passive elements for presbyopia compensation as multifocal lenses $[14,15,16]$ and elements focusing light into line segments (LSOE, axicons) [5, 17, 18, 19]. Therefore besides the LSOE we analyze for comparison the imaging properties of a bifocal lens and an inverse quartic axicon. 


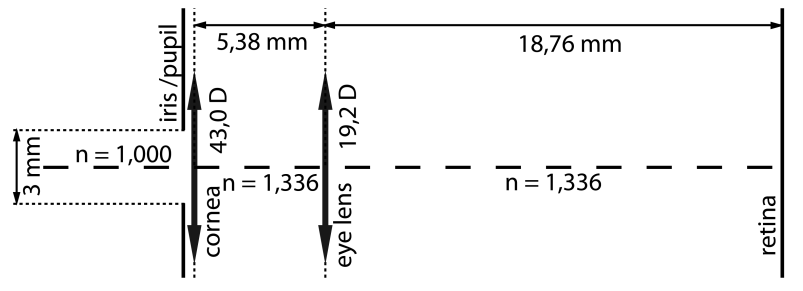

FIC. 1 A scheme of an optical set-up based on the Cullstrand model of the relaxed human eye with a reduced aperture of diameter $3 \mathrm{~mm}$. Analyzed compensating elements are located as contact lenses in the pupil plane.

\section{EYE MODEL AND COMPENSATING ELEMENTS}

We restrict our investigations to the small foveal region of the retina where vision is most acute. This assumption makes possible to assume the paraxial approximation and isoplanarity of an imaging system in calculations of light propagation and during the design of optical elements for presbyopia compensation. According to this approach the Gullstrand model of the presbyopic eye can be simplified to that shown in Figure 1 [6]. The cornea and the eye lens are substituted by thin lenses with powers $43 \mathrm{D}$ and 19.2 $\mathrm{D}$ respectively, which are located at mutual distance $5.38 \mathrm{~mm}$. The output plane corresponding to the retina is situated at a distance $18.76 \mathrm{~mm}$ behind the second lens. The optical system is immersed in a liquid with a refractive index equal to 1.336. Additionally we assume that the system is limited by a pupil of diameter $3 \mathrm{~mm}$ placed in the plane of the lens representing the cornea. This dimension of the iris ensures satisfactory resolution of photopic vision [11]. In the same plane there can be inserted a proper element designed for presbyopia correction as a contact lens. The arrangement in Figure 1 represents the reference case of the naked presbyopic eye (EYE) that can realize acute vision for objects situated at infinity. In the paper we analyze the imaging properties of this system together with five different optical arrangements simulating the EYE corrected by the following elements:

\subsection{Reading glasses (RG)}

The naked presbyopic eye is completed by a convergent lens with power $4 \mathrm{D}$ designed for input objects located at a distance $25 \mathrm{~cm}$.

\subsection{Bifocal contact lens (BFCL)}

As a compensating element is implemented an annularly oriented bifocal contact lens used in Acuvue Bifocal contact lenses [20]. The lens has two values of optical powers equal to $0 \mathrm{D}$ or $4 \mathrm{D}$ for ranges of radii [0 $\mathrm{mm}, 1.08 \mathrm{~mm}$ ] and [1.08 $\mathrm{mm}, 1.5 \mathrm{~mm}$ ] respectively.

\subsection{Inverse quartic axicon (IQAX)}

The IQAX represents here axicons, elements with revolution symmetry being modified spherical lenses with controlled spherical aberrations. The IQAX is a typical structure of this kind designed for imaging with an extended depth of field (EDOF). It exhibits optical properties similar to the logarith-
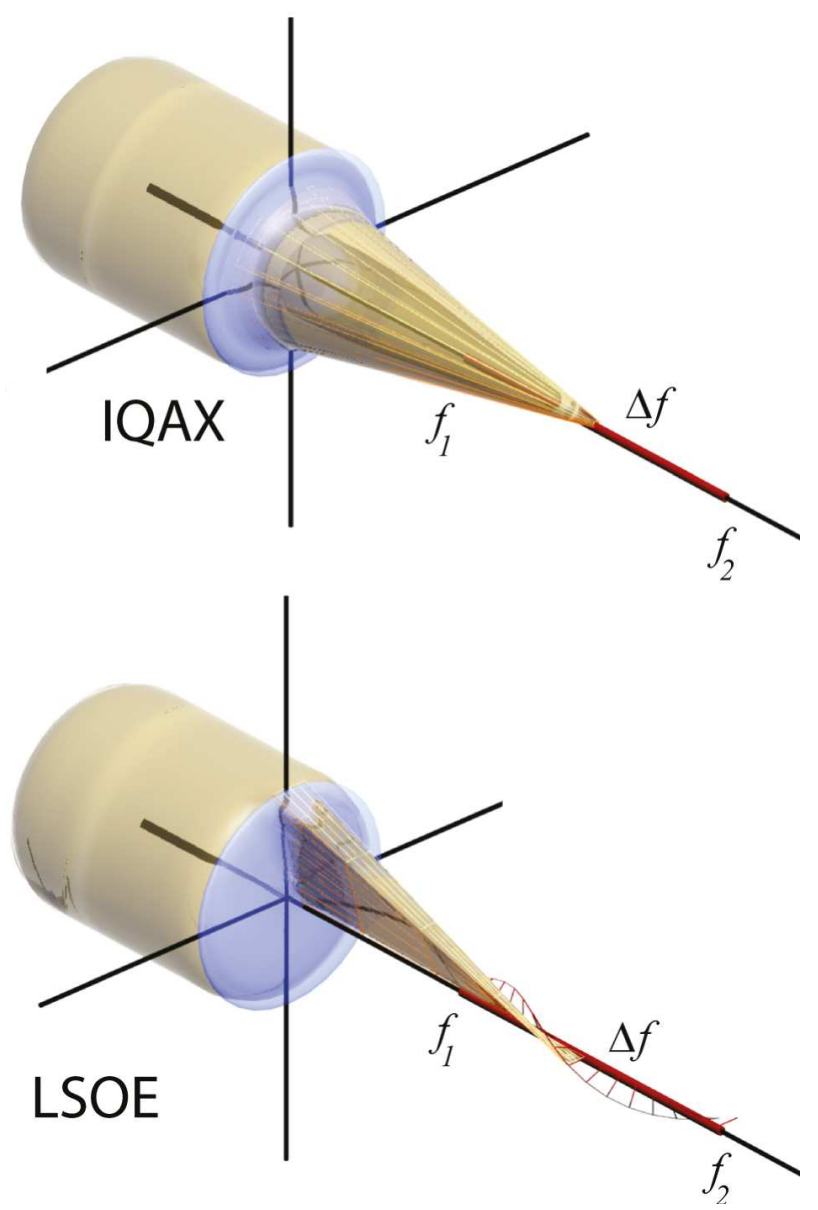

FIG. 2 Geometry of focusing corresponding to the IQAX and the LSOE according to geometrical optics.

mic axicon [17] and can be potentially useful for presbyopia correction [19]. According to geometrical optics the IQAX focuses an incident plane wave into a line segment stretched from a distance $f_{1}$ up to a distance $f_{2}=f_{1}+\Delta f$ behind the element. Geometry of focusing is shown in Figure 2 where an infinitesimal annular part of the structure focuses light in a proper point of the focal segment. The optical path difference (OPD) caused by the IQAX with an aperture radius $R$ is given as follows:

$$
\begin{gathered}
\Delta l(r)=A r^{2}+B r^{4} \\
A=-\frac{1}{2\left(f_{1}+\Delta f\right)}, B=-\frac{1}{4 R^{2}}\left(\frac{1}{f_{1}}-\frac{1}{f_{1}+\Delta f}\right),
\end{gathered}
$$

where $r$ is the radial coordinate in the polar coordinate system.

\subsection{Light sword optical element (LSOE)}

The LSOE is an imaging element distinguished by an angular modulation [5]. It realizes the following OPD:

$$
\Delta l(r, \theta)=-\frac{r^{2}}{2\left(f_{1}+\frac{\Delta f \theta}{2 \pi}\right)},
$$

where $\theta$ is the azimuthal coordinate in the polar coordinate system. Therefore according to Eq. (2) an infinitesimal angular sector of the LSOE is equivalent to a spherical convergent lens with focal length $f_{1}+\Delta f \theta / 2 \pi$. Then in an approximation this element focuses a plane wave into the segment presented 
in Figure 2. A more detailed analysis based on the ray tracing method leads to the conclusion that otherwise to axicons focusing is not perfect [21]. Each angular sector of the structure focuses light into a perpendicularly oriented small strip. Nevertheless, as it was lately proved both numerically and experimentally, the LSOE manifests excellent abilities for EDOF imaging $[6,21,22,23]$.

\subsection{Additive light sword optical element (aLSOE)}

In a sense of the design, the aLSOE is a counterpart of the IQAX with introduced angular aberrations. The OPD corresponding to the element is described by the following equation:

$$
\begin{gathered}
\Delta l(r, \theta)=C r^{2}+D \theta r^{2}, \\
C=-\frac{1}{2 f_{1}}, D=\frac{1}{4 \pi}\left(\frac{1}{f_{1}}-\frac{1}{f_{1}+\Delta f}\right) .
\end{gathered}
$$

The geometry of focusing by the aLSOE is analogous to that shown in Figure 2 for the LSOE.

The last three elements (IQAX, LSOE, aLSOE) realizing EDOF imaging and analyzed in the paper work as contact lenses for EYE shown in Figure 1. Thus the elements are properly designed to compensate defocusing from $0 \mathrm{D}$ up to $4 \mathrm{D}$ that corresponds to object distances covering a range $[25 \mathrm{~cm}, \infty)$. Figure 3 presents the optical power distribution within the studied five elements compensating EYE. According to our assumptions the structures have aperture diameters reduced to $3 \mathrm{~mm}$.

\section{NUMERICAL SIMULATIONS AND OBTAINED RESULTS}

We analyze the contrast transfer in considered arrangements by means of MTFs and Siemens star images. The MTF gives a global outlook in imaging [24]. MTFs values are equal to the contrast (visibility) of fringes being an image of a sinusoidal grating defined by a proper spatial frequency. Therefore the MTF enables to predict qualities of output image fragments corresponding to known spatial frequencies in the object plane. On the other hand the Siemens star can be locally regarded as a Ronchi grating with a defined orientation of fringes then the quality of its image is strictly connected to the adequate domains of the MTF. Hence a MTF and a Siemens star image are mutually related [24] and supplement each other.

Our numerical simulations are conducted by means of a software package based on the modified convolution method $[12,13]$. We assume spatially incoherent light illumination of a wavelength $555 \mathrm{~nm}$ corresponding to the highest sensitivity of the human eye in photopic vision [11]. For a fixed object distance a point image is calculated in the setup shown in Figure 1 with a proper compensating element. The entrance aperture is reduced to $3 \mathrm{~mm}$ and thin lenses representing the cornea and the human eye's lens are immersed in a medium with a refractive index equal to 1.336. Additionally we take into account the Stiles-Crawford effect. Then the light ampli-
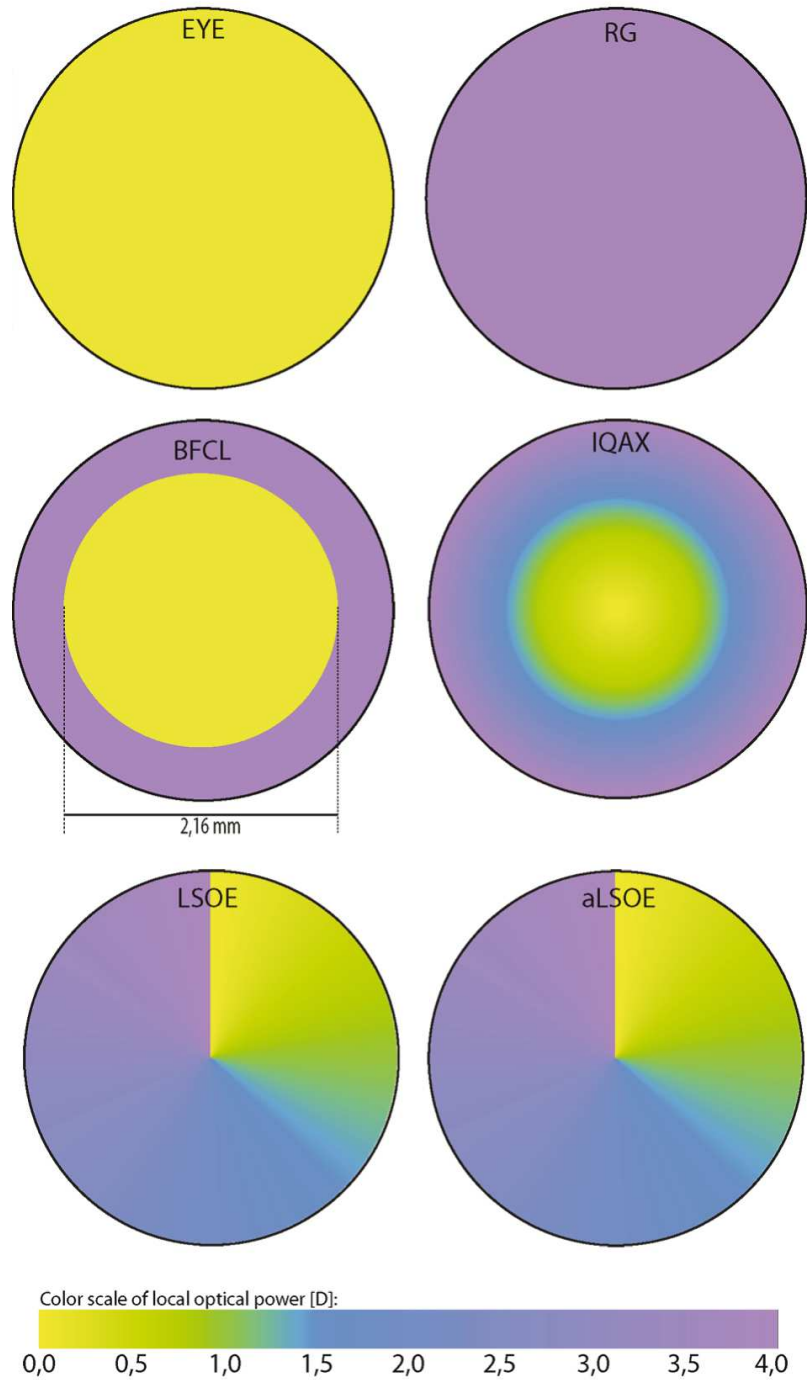

FIG. 3 Optical compensating elements used in simulations. Their diameter was assumed to be equal to $3 \mathrm{~mm}$. The used abbreviations are explained in the text. The optical power corresponding to different areas is marked with the color bar.

tude behind the cornea is multiplied by an exponential factor equal to $\exp \left(0.053 r^{2}\right)$, where $r$ is expressed in mm [11]. After propagation in the medium we obtain the output amplitude in the retinal plane. The square modulus of the amplitude defines the point spread function (PSF) of an imaging system. The MTF is calculated by means of the PSF's Fourier transform. Taking into account the system's isoplanarity, output images are equivalent to the convolution of the ideal geometric image with the PSF. The numerical approach is illustrated in Figure 4.

The MTF has point symmetry [24], hence we show only the upper half of the Siemens star images. The star resolution test used in this work is consisted of 48 arms being periodically transparent and opaque. Figure 5 presents the output ideal image of the star demagnified in a set-up simulating the human eye (Figure 1) and formed in a central region of the retina with dimensions $0.30 \mathrm{~mm} \times 0.15 \mathrm{~mm}$. For all defocusing parameters we assume constant angular dimensions of input objects. Then the obtained results are characterized by angular frequencies measured in lines per degree ( $1 / \mathrm{deg})$. Red semicircles in Figure 5 indicate points corresponding to local fre- 


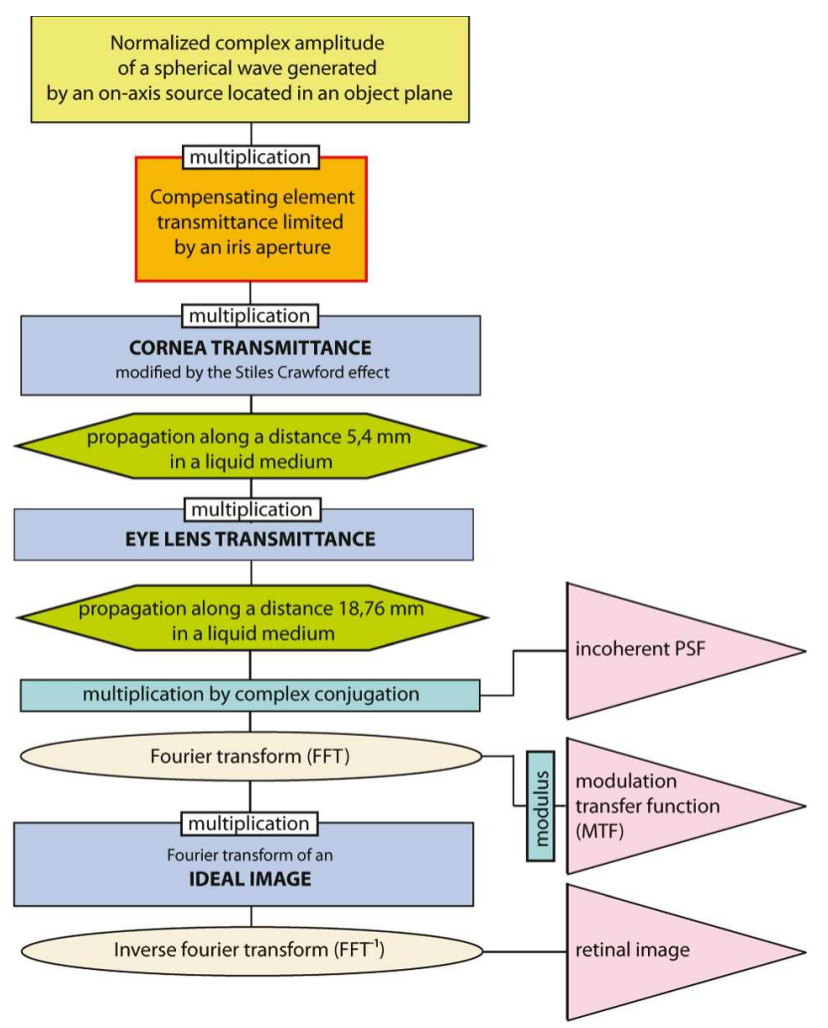

FIG. 4 A scheme of numerical simulations.

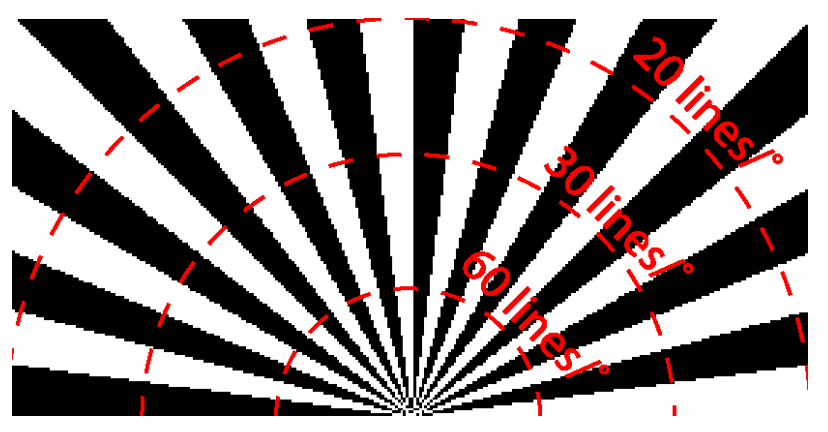

FIG. 5 Ideal image of the Siemens star obtained in a central fragment of the imaging plane with dimensions $0.30 \mathrm{~mm} \times 0.15 \mathrm{~mm}$.

quencies 60, 30 and 20 1/deg respectively. Figure 6, Figure 7 and Figure 8 show output images created in the arrangement presented in Figure 1 without (EYE) and with compensating elements (RG, BFCL, IQAX, LSOE, aLSOE). Results have been simulated for the following seven values of defocus: 4, 3.34, $2.67,2,1.34,0.67,0$ dioptres, which correspond to object distances: $25 \mathrm{~cm}, 30 \mathrm{~cm}, 37 \mathrm{~cm}, 50 \mathrm{~cm}, 75 \mathrm{~cm}, 150 \mathrm{~cm}$ and $\infty$, respectively. Yellow semicircles determine angular frequencies in an ideal geometric image and orange percentages describe the local fringes' contrast connected to the above frequencies in the output plane simulating the retina. The contrast is calculated by minimal and maximal local intensities in the following way: $\pm 100 \%\left(I_{\max }-I_{\min }\right) /\left(I_{\max }+I_{\min }\right)$. A negative value denotes a contrast reversal.

MTFs calculated for the same arrangements and object distances complement the images of the star resolution test. Figure 9, Figure 10 and Figure 11 illustrate MTFs bitmaps with white or black semicircles indicating proper angular frequencies. Due to our short discussion the results presented in Figure 6, Figure 9, Figure 7, Figure 10 and Figure 8, Figure 11 co-

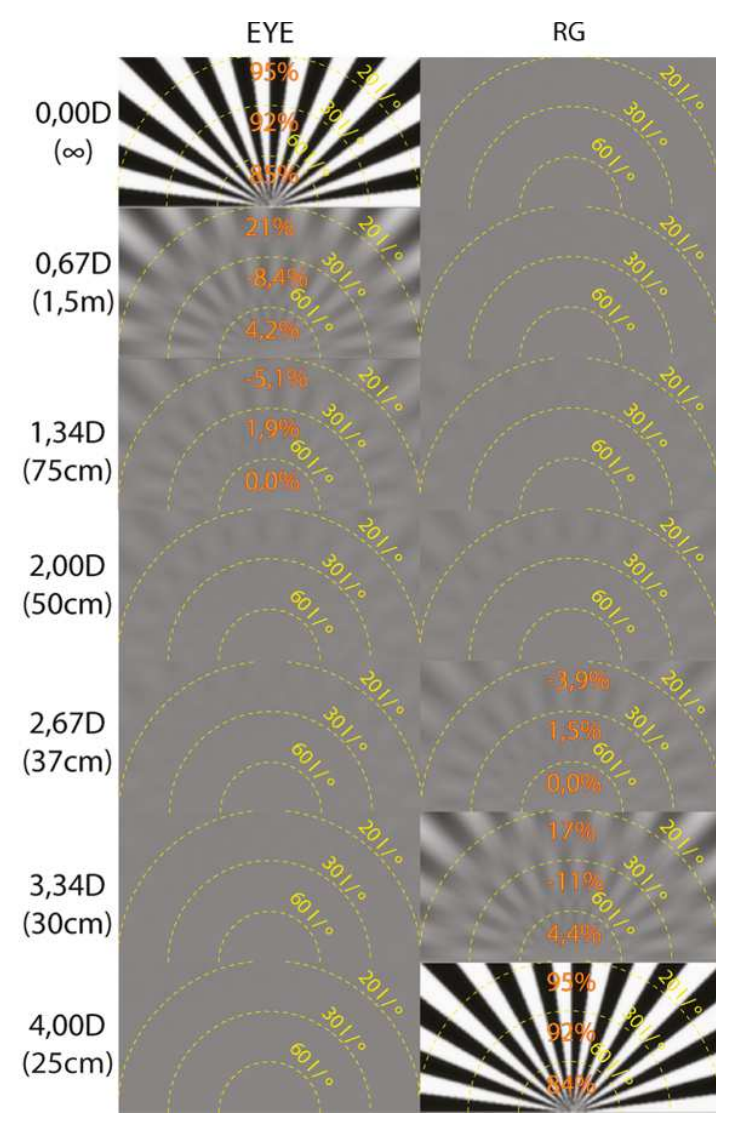

FIG. 6 Images of the star resolution test before (EYE) and after correction by the RG. Defocus powers and relative object distances are given in the left side. Other notations are explained in the text.

incide and they lead to similar conclusions. The EYE and the RG operate excellently as diffraction limited systems for far and near vision respectively. However, even a small defocusing about $0.6 \mathrm{D}$ spoils completely the output image. A contrast reversal appears and the MTF has wide pass-bands with zero values. The BFCL with optical powers $4 \mathrm{D}$ and $0 \mathrm{D}$ forms satisfactory images of objects located at designed distances $25 \mathrm{~cm}$ and $\infty$. MTFs demonstrate in these cases pass-bands reaching 70-94 1/deg. The BFCL manifests abilities for imaging with a reduced EDOF corresponding to far object distances. Nevertheless, defocusing causes significant degradation of output images. Therefore the Siemens star is not recognizable for values of intermediate defocus. The IQAX designed for all object distances from $25 \mathrm{~cm}$ fulfills its task only partly. Moderate defocus up to $1.34 \mathrm{D}$ connected to far vision does not substantially modify the quality of imaging. The contrast is quite acceptable and there are wide ranges of frequencies where the MTFs have nonzero values. The situation changes dramatically in the case of higher defocus. MTFs exhibit strong oscillations with zero values. Central nonzero parts correspond only to small frequencies. Therefore a contrast reversal occurs and the output images are completely blurred. Hence the use of the IQAX seems to be strongly reduced in a case of near vision.

According to Figure 8 and Figure 11 the LSOE and the aLSOE manifest original but interesting imaging properties. The results obtained for both elements are very similar. The MTFs have nonzero values for wide ranges of frequencies. Simultaneously the MTFs do not exhibit substantial oscillations. On 


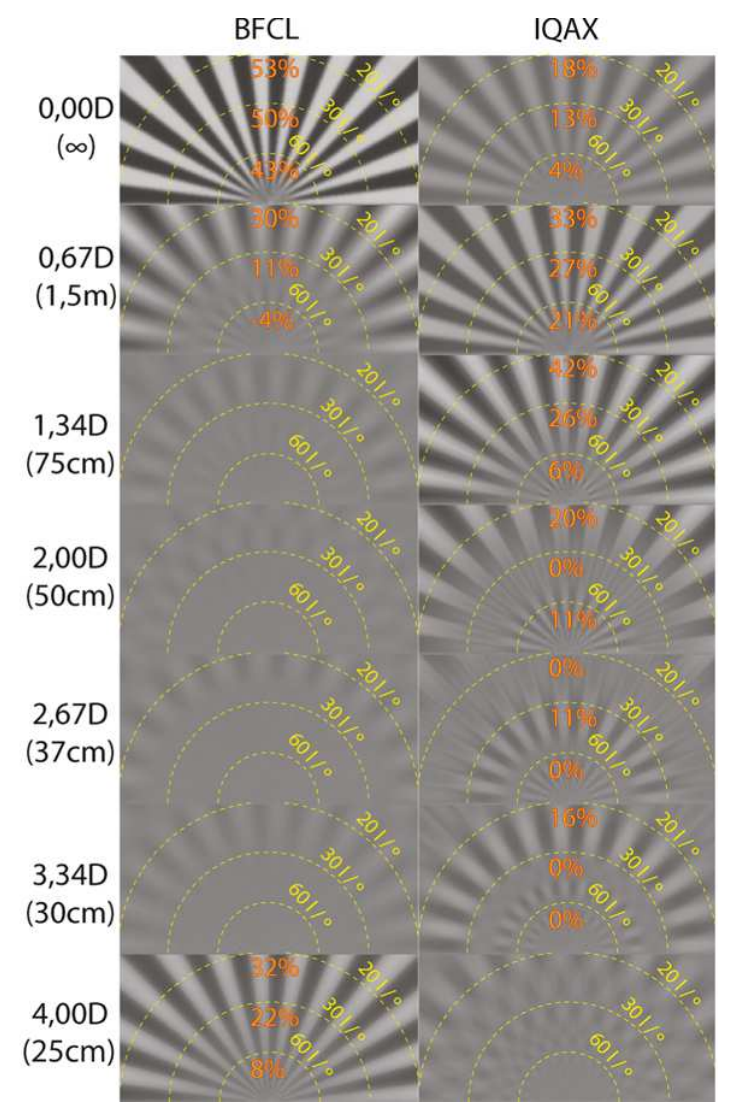

FIC. 7 Images of the star resolution test after correction by the BFCL and the IQAX. Defocus powers and relative object distances are given in the left side. Other notations are explained in the text.

the other hand the MTFs have not got symmetry of revolution and they illustrate well the directional properties of imaging realized by the LSOE. This effect is visible in Figure 8 . The local contrast of the output fringes depends not only on the magnitudes of the angular frequencies but also on their directions. Therefore in cases of the LSOE and the aLSOE the contrast shown in Figure 8 corresponds to maximal values calculated for presented yellow semicircles. This directional dependence spoils slightly the output images of the star resolution test. Nevertheless, fringes are easily recognizable for all assumed object distances and the indicated maximal contrasts have acceptable positive values, substantially greater than zero. Hence the LSOE and the aLSOE can successfully realize EDOF imaging within the range of assumed defocus up to $4 \mathrm{D}$.

\section{Conclusions.}

The performed simulations prove that the LSOE and the aLSOE exhibit better abilities for EDOF imaging in ophthalmical applications than other hitherto known solutions. Both elements easily compensate defocus covering object distances within the range $[25 \mathrm{~cm},+\infty)$. The application of the LSOE and the aLSOE causes a characteristic haze but output images are well defined. According to Figure 8 the contrast reversal usually being responsible for a substantial image degradation does not appear in the present case. Contrary to LSOEs the IQAX realizes a limited depth of focus although the axicon was designed for the same object distances. Figure 7 presents

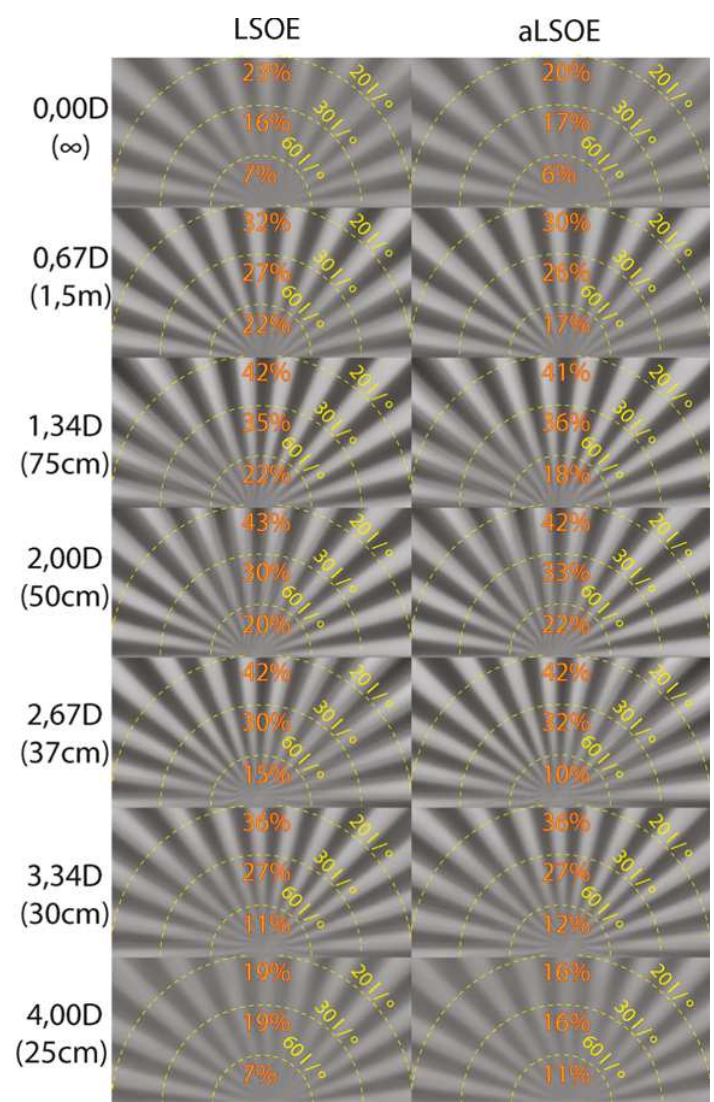

FIG. 8 Images of the star resolution test after correction by the LSOE and the aLSOE. Defocus powers and relative object distances are given in the left side. Other notations are explained in the text.

a satisfactory correction by the IQAX for far vision distances only. The RG and the BFCL reveal imaging properties being consistent with expectations. The output images have acceptable qualities only for object distances matched for those assumed during the design. The above remarks regarding the five analyzed elements coincide well with conclusions given in our paper discussing Strehl ratios [6] and are supported by experimental results using natural images which are subject of a separate forthcoming publication in order to avoid excessive length of the present paper.

In spite of good possibilities for defocus compensation LSOEs exhibit some disadvantages. According to the ray tracing method infinitesimal angular sectors of an element focus an incident plane wave in a small light segment instead of a point. Hence PSFs formed by LSOEs demonstrate a characteristic smear leading to glare and haze effects in the output images. Additionally they wave slightly around the optical axis what results in the image displacement [23]. On the other hand this geometry of focusing discussed in Ref. [21] converts into a substantial advantage during EDOF imaging. The process of focusing has an off-axis character. As it is illustrated by ray tracing in Figure 2, the flow of energy changes its main direction during focusing by the LSOE. Then an effect of mutual disturbance between neighboring images corresponding to different focal length or object distances is less harmful than in cases of axicon with a radial symmetry, where a flow of energy during focusing has the same direction along the optical axis. Additionally it is worth to note that the refractive version of the LSOE does not exhibit noticeable chromatic aberrations 


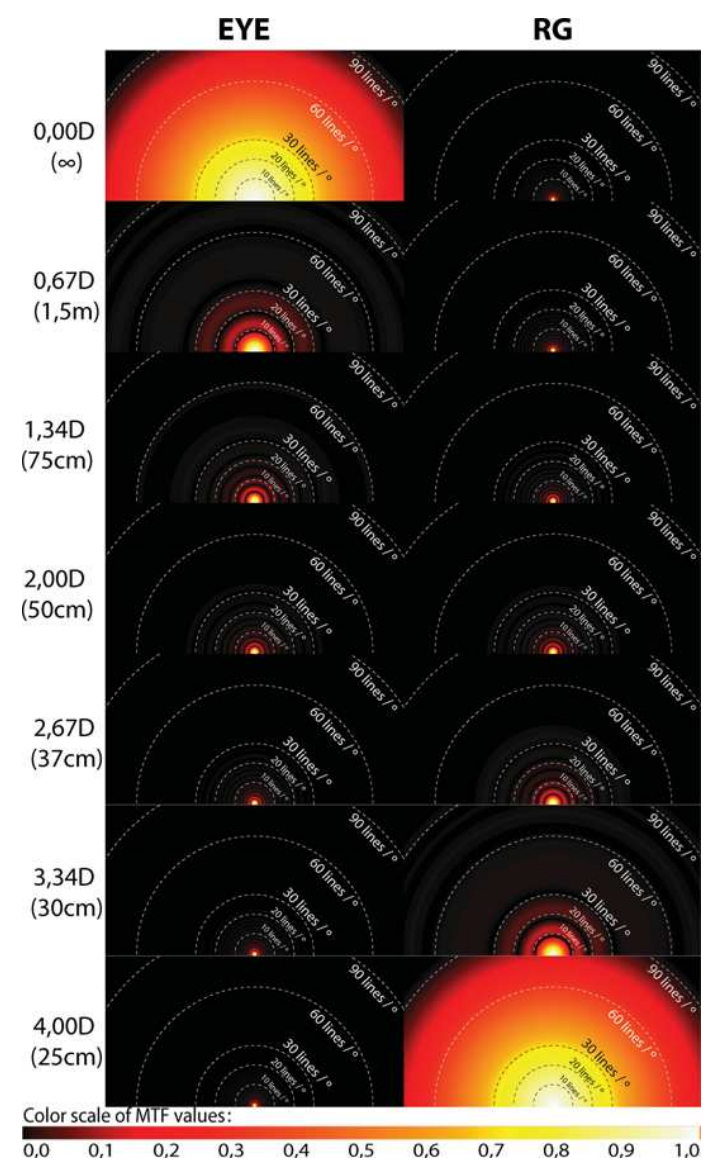

FIG. 9 MTF functions of the EYE and the RG related to defocusing illustrated in Figure 6. Notations are explained in the text.

[22]. Moreover, according to the geometry of focusing (Figure 2) the focal range of the LSOE is independent of the aperture diameter otherwise to elements where the optical power is radially distributed [25].

Generally, the presented results lead to the conclusions that the LSOE and the aLSOE seem to be very promising alternative for compensation of presbyopia of the human eye. Nevertheless, the inaccuracy of light focusing and the directional character of imaging illustrated by the MTFs in Figure 11 cause a degradation in the output images formed by the LSOEs. One can hope that this inconvenience can be at least partly compensated by the brain image processing procedures and a psychophysical adaptability of the human eye for the LSOEs aberrations is possible [26]. These important problems are out of scope of this paper and they need further studies and clinical tests.

\section{Acknowledgments}

This work was supported by the Polish Ministry of Science and Higher Education under grant N N 514149038 and by the Spanish Ministerio de Ciencia e Innovacion (MICINN), grant FIS2008-03884 with complementary support from the European Social Fund implemented under the Human Capital Programme (POKL), project: "Preparation and Realization of Medical Physics Specialty". A. Ciro Lopez wants to give his thanks to Instituto Tecnologico Metropolitano de Medellin by

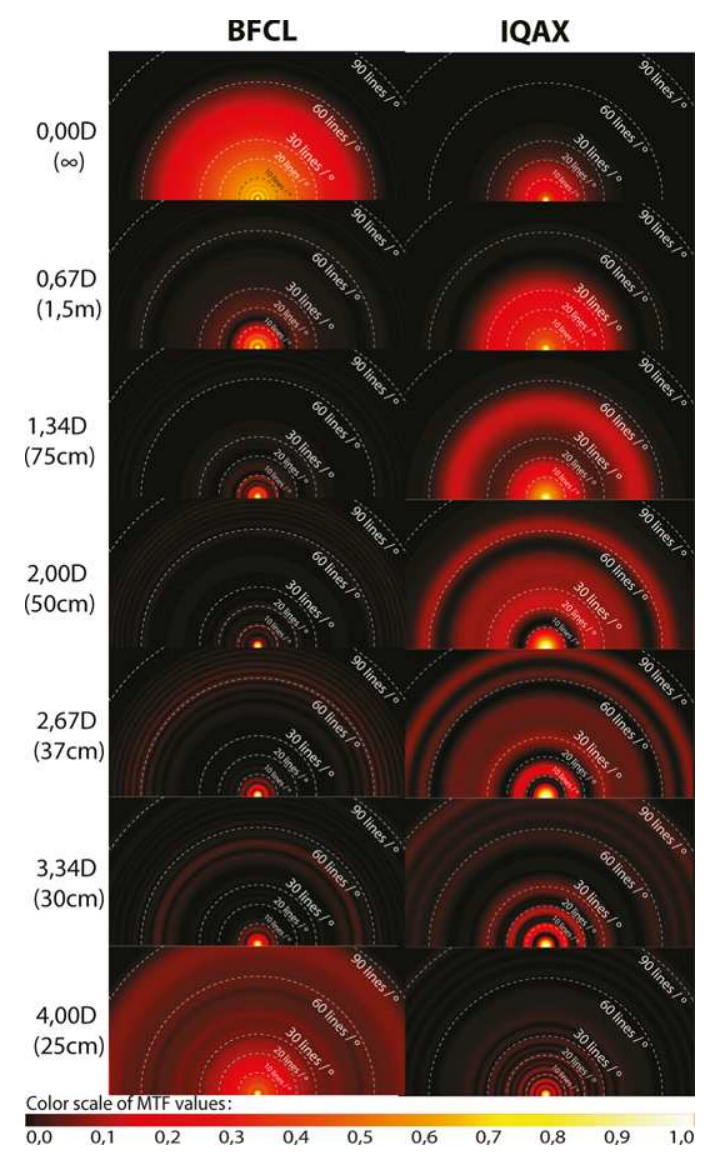

FIG. 10 MTF functions of the BFCL and the IQAX related to defocusing illustrated in Figure 7 . Notations are explained in the text.

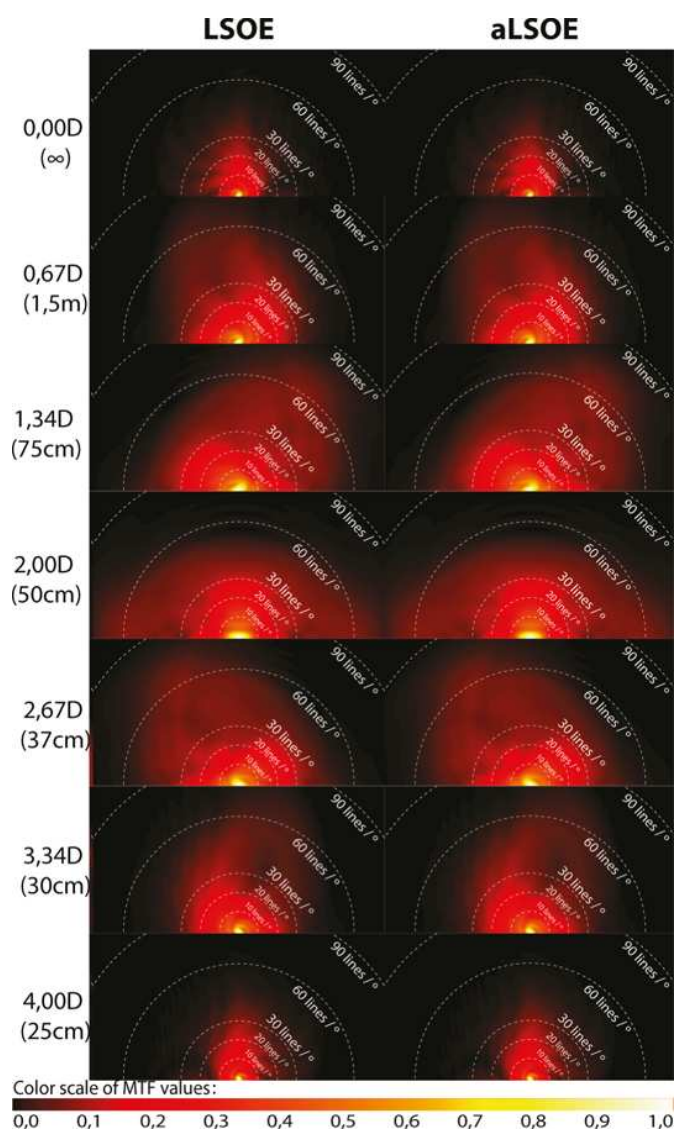

FIG. 11 MTF functions of the LSOE and the aLSOE related to defocusing illustrated in Figure 8. Notations are explained in the text. 
valuable support and facilitating the necessary labor time for participation in this work.

\section{References}

[1] P. Artal, and J. Tabernero, "Optics of human eye: 400 years of exploration from Galileo's time" Appl. Opt. 49, D123-D130 (2010).

[2] B. K. Pierscionek, "What we know and understand about presbyopia" Clin. Exp. Optom. 76, 83-90 (1993).

[3] A. Glasser, M. A. Croft, and P. L. Kaufman, "Aging of the Human Crystalline Lens and Presbyopia" Int. Ophthalmol. Clin. 41, 1-15 (2001).

[4] Z. Zalevsky, "Extended depth of focus imaging: a review" SPIE Reviews 1, 018001 1-11 (2010).

[5] A. Kolodziejczyk, S. Bara, Z. Jaroszewicz, and M. Sypek, "The light sword optical element - a new diffraction structure with extended depth of focus" J. Mod. Opt. 37, 1283-1286 (1990).

[6] K. Petelczyc, J. Ares Garcia, S. Bara, Z. Jaroszewicz, K. Kakarenko, A. Kolodziejczyk, and M. Sypek, "Strehl ratios characterizing optical elements designed for presbyopia compensation" Opt. Express 19, 8693-8699 (2011).

[7] J. D. Marsack, L. N. Thibos, and R. A. Applegate, "Metrics of optical quality derived from wave aberrations predict visual performance" J. Vision 4, 322-328 (2004).

[8] R. D. Iskander, "Computational aspects of the visual Strehl ratio" Optometry Vision Sci. 83, 57-59 (2006).

[9] G.-M. Dai, "Optical surface optimization for the correction of presbyopia" Appl. Opt. 45, 4184-4195 (2006).

[10] A. Valberg, Light Vision Color (John Wiley đt Sons, New York, 2005).

[11] H. Gross, F. Blechinger, and B. Achtner, Handbook of Optical Systems, Vol. 4, Survey of Optical Instruments (Wiley-VCH, Weinheim, 2008).

[12] M. Sypek, "Light propagation in the Fresnel region. New numerical approach" Opt. Commun. 116, 43-48 (1995).

[13] M. Sypek, C. Prokopowicz, and M. Gorecki, "Image multiplying and high-frequency oscillations effects in the Fresnel region light propagation simulation" Opt. Eng. 42, 3158-3164 (2003).

[14] J. A. Davison, and M. J. Simpson, "History and development of the apodized diffractive intraocular lens" J. Cataract. Refr. Surg. 32, 849-858 (2006).

[15] B. Żelichowska, M. Rekas, A. Stankiewicz, A. Cerviño, R. MontesMico, "Apodized diffractive versus refractive multifocal intraocular lenses: Optical and visual evaluation" J. Cataract. Refr. Surg. 34, 2036-2042 (2008).

[16] P. J. Valle, J. E. Oti, V. F. Canales, and M. P. Cagigal, "Visual axial PSF of diffractive trifocal lenses" 0pt. Express 13, 2782-2792 (2005).

[17] J. Sochacki, A. Kolodziejczyk, Z. Jaroszewicz, and S. Bara, "Nonparaxial design of generalized axicons" Appl. Opt. 31, 5326-5330 (1992).

[18] W. Chi, and N. George, "Electronic imaging using a logarithmic asphere" 0pt. Lett. 26, 875-877 (2001).

[19] J. Ares, R. Flores, S. Bara, and Z. Jaroszewicz, "Presbyopia compensation with a quartic axicon" Optometry Vision Sci. 82, 1071-1078 (2005).

[20] J. H. Roffman, T. R. Poling, and M. Guillon, "Pupil-tuned multifocal ophthalmic lens" US Patent 5,448,312 (1995).
[21] G. Mikula, Z. Jaroszewicz, A. Kolodziejczyk, K. Petelczyc, and M. Sypek, "Imaging with extended focal depth by means of lenses with radial and angular modulation" Opt. Express 15, 9184-9193 (2007).

[22] J. Ares Garcia, S. Bará, M. Gomez García, Z. Jaroszewicz, A. Kolodziejczyk, and K. Petelczyc, "Imaging with extended focal depth by means of the refractive light sword optical element" Opt. Express 16, 18371-18378 (2008).

[23] G. Mikula, A. Kolodziejczyk, M. Makowski, C. Prokopowicz, and M. Sypek, "Diffractive elements for imaging with extended depth of focus" Opt. Eng. 44, 058001 1-7 (2005).

[24] J. W. Goodman, Introduction to Fourier Optics (Roberts \& Company Publishers, Greenwood Village, 2005).

[25] K. Petelczyc, J. Ares Garcia, S. Bará, Z. Jaroszewicz, A. Kolodziejczyk, and M. Sypek, "Presbyopia compensation with a light sword optical element of a variable diameter" Phot. Lett. Poland 1, 55-57 (2009).

[26] P. Artal, L. Chen, E. J. Fernandez, B. Singer, S. Manzanera, and D. R. Williams, "Neural compensation for the eye's optical aberrations" J. Vision 4, 281-287 (2004). 